\title{
BestMasters
}


Mit „BestMaster““ zeichnet Springer die besten Masterarbeiten aus, die an renommierten Hochschulen in Deutschland, Österreich und der Schweiz entstanden sind. Die mit Höchstnote ausgezeichneten Arbeiten wurden durch Gutachter zur Veröffentlichung empfohlen und behandeln aktuelle Themen aus unterschiedlichen Fachgebieten der Naturwissenschaften, Psychologie, Technik und Wirtschaftswissenschaften.

Die Reihe wendet sich an Praktiker und Wissenschaftler gleichermaßen und soll insbesondere auch Nachwuchswissenschaftlern Orientierung geben. 
Martin Egeli

\section{Erfolgsfaktoren von Mobile Business}

Ein Reifegradmodell zur digitalen Transformation von Unternehmen durch Mobile IT

Mit einem Geleitwort von Prof. Dr. Andrea Back

블 Springer Vieweg 
Martin Egeli

Zürich, Schweiz

Originaltitel der Masterarbeit: Mobile Business Maturity Model: Entwicklung eines Reifegradmodells zur digitalen Transformation von Unternehmen durch Mobile IT

OnlinePLUS Material zu diesem Buch finden Sie auf

http://www.springer-vieweg.de/978-3-658-12770-1

\section{BestMasters}

ISBN 978-3-658-12770-1

ISBN 978-3-658-12771-8 (eBook)

DOI 10.1007/978-3-658-12771-8

Die Deutsche Nationalbibliothek verzeichnet diese Publikation in der Deutschen Nationalbibliografie; detaillierte bibliografische Daten sind im Internet über http://dnb.d-nb.de abrufbar.

Springer Vieweg

(C) Springer Fachmedien Wiesbaden 2016

Das Werk einschließlich aller seiner Teile ist urheberrechtlich geschützt. Jede Verwertung, die nicht ausdrücklich vom Urheberrechtsgesetz zugelassen ist, bedarf der vorherigen Zustimmung des Verlags. Das gilt insbesondere für Vervielfältigungen, Bearbeitungen, Übersetzungen, Mikroverfilmungen und die Einspeicherung und Verarbeitung in elektronischen Systemen.

Die Wiedergabe von Gebrauchsnamen, Handelsnamen, Warenbezeichnungen usw. in diesem Werk berechtigt auch ohne besondere Kennzeichnung nicht zu der Annahme, dass solche Namen im Sinne der Warenzeichen- und Markenschutz-Gesetzgebung als frei zu betrachten wären und daher von jedermann benutzt werden dürften.

Der Verlag, die Autoren und die Herausgeber gehen davon aus, dass die Angaben und Informationen in diesem Werk zum Zeitpunkt der Veröffentlichung vollständig und korrekt sind. Weder der Verlag noch die Autoren oder die Herausgeber übernehmen, ausdrücklich oder implizit, Gewähr für den Inhalt des Werkes, etwaige Fehler oder Äußerungen.

Gedruckt auf säurefreiem und chlorfrei gebleichtem Papier

Springer Vieweg ist Teil von Springer Nature

Die eingetragene Gesellschaft ist Springer Fachmedien Wiesbaden GmbH 


\section{Geleitwort}

Die Digitale Transformation ist heute eine allseits wahrgenommene Entwicklung. Der Handlungsbedarf auf allen Ebenen - wirtschaftlich, politisch und persönlich - ist anerkannt. „Alles wird digital sein“, lassen Trendforscher verlauten, und oft liest man zusätzlich: "Alles was digital ist, wird mobil sein“. Vor diesem Hintergrund befasst sich die Arbeit von Martin Egeli mit der digitalen Transformation von Unternehmen durch Mobile Informationstechnik (IT). Mit seinem Reifegradmodell liegt ein einfach verständliches und allgemein praktikables Managementinstrument vor, das Unternehmen und andere Organisationen nutzen können, um den eigenen Entwicklungsstand zu reflektieren und Weiterentwicklungen anzustossen.

Reifegradmodelle sind ein vielseitig einsetzbares Managementinstrument und deshalb auch in der Beratung verbreitet. Je nach Nutzungskontext können sie als Gesprächsgrundlage in gemischten Managementteams dienen, als SelfAssessment Lücken aufzeigen und zur Fokussierung von Investitionen beigezogen werden. Eine weitgehendere Nutzung ist, sie zum periodischen Monitoring der eigenen Weiterentwicklung einzusetzen.

Das von Egeli fundiert entwickelte Mobile Business Maturity Model lehnt sich in seiner Struktur an die bewährte St. Galler Business Engineering-Landkarte an, die auch das Rückgrat des schon viele Jahrgänge umfassenden Executive Master of Business Engineering bildet, d.h. sich in der Praxis-Community etabliert und bewährt hat. Auch Egeli hat mehrere Praxisexperten einbezogen, um sein Modell sogleich wissenschaftlich hergeleitet und praxisnah einsetzbar zu gestalten.

Die Masterarbeit zu diesem aktuellen Thema zu publizieren und damit einer interessierten Leserschaft zugänglich zu machen, lag auf der Hand, zumal auch ein Excel-basiertes Assessment-Werkzeug vorliegt, mit dem unmittelbar 
gearbeitet werden kann. Dass Springer mit der Reihe BestMasters die vollständige Veröffentlichung als Buch ermöglicht, schätzen wir sehr. Wir wünschen uns, dass daraus ein Beispiel für einen gelungenen Wissenstransfer zwischen Hochschule und Praxis wird; ganz im Sinne des Anspruchs der Universität St. Gallen: „Wissen schafft Wirkung“.

Prof. Dr. Andrea Back

Institut für Wirtschaftsinformatik

Universität St. Gallen (HSG), Schweiz 


\section{Institutsprofil}

Die Arbeit von Martin Egeli ist am Institut für Wirtschaftsinformatik der Universität St. Gallen entstanden. Sie wurde am Lehrstuhl von Prof. Dr. Andrea Back von Sabine Berghaus, Projektleiterin in unserem Arbeitsschwerpunkt Competence Center (CC) Mobile Business, betreut.

Im CC Mobile Business beschäftigen wir uns mit der Frage, wie mobile Technologien innovativ im Unternehmenskontext genutzt werden können. Wir pflegen neben der akademischen Spitzenforschung, die in JournalPublikationen mündet, einen intensiven Austausch mit Praxispartnern, auch in der Lehre. So sind im Fachbuch Sammer/Back/Walter: Mobile Business - Management von Mobiler IT in Unternehmen mehrere Unternehmensfallstudien mit Video-Interviews enthalten. Die aktive Zusammenarbeit mündet in anwendungsorientierten Forschungsprojekten mit dem Ziel der Wirtschaftsförderung, in Whitepapers und Marktstudien u.a.m. Das jährliche St. Gallen Mobile Business Forum, an dem auch der Mobile Business Best Practice Award verliehen wird, ist eine Plattform für den Dialog von Wissenschaft, Unternehmenspraxis sowie Start-ups, und thematisiert vorausschauend die nächsten Innovationsstufen von Mobile Business. 


\section{Abstract}

Die vorliegende Arbeit befasst sich mit der digitalen Transformation von Unternehmen durch Mobile IT und entwickelt dazu ein Maturity Model. Dieses Reifegradmodell soll Unternehmen dabei unterstützen, eine Standortbestimmung vornehmen zu können, um so die eigene Reife im professionellen Umgang mit Mobile Business zu erkennen und daraus Möglichkeiten für Weiterentwicklungen und Verbesserungen im Unternehmen abzuleiten. Zur Erarbeitung der Modellinhalte werden 20 bestehende Maturity Models zum Themengebiet identifiziert sowie weitere Studien und wissenschaftliche Arbeiten zu Mobile Business herangezogen. Das damit eigenständig konstruierte Maturity Model wird anschliessend anhand von sechs Experteninterviews mit Unternehmensvertretern aus der Schweiz und Deutschland evaluiert und vervollständigt.

Das entwickelte Mobile Business Maturity Model lehnt sich strukturell an der St. Galler Business Engineering-Landkarte an: In dessen vier Ebenen Strategie, Prozesse, Systeme und Kultur sind insgesamt zehn Gestaltungsdimensionen des Modells gruppiert, welche unterschiedliche thematische Aspekte rund um Mobile Business adressieren. Jeweils drei Gestaltungsobjekte pro Dimension dienen als Beurteilungskriterien um die Reife in jeder Dimension zu bestimmen. Daraus wird einer von fünf Gesamtreifegraden ermittelt, welcher ausdrücken soll, wie professionell ein Unternehmen mit Mobile Business umgeht: Geringe Mobilität (Reifegrad 1), reaktive Mobilität (2), situative Mobilität (3), strategische Mobilität (4) und integrale Mobilität (5). Um interessierten Kreisen eine einfache Anwendung des Maturity Models zu ermöglichen, wurde als weiteres Ergebnis der Arbeit ein Excel-basiertes Assessment-Werkzeug entwickelt, das direkt von http://fyayc.com/mobile-maturity oder auf der Produktseite des Buches unter www.springer.com heruntergeladen werden kann. 


\section{Inhaltsverzeichnis}

Abbildungsverzeichnis ............................................................................... XV

Tabellenverzeichnis ......................................................................................

Abkürzungsverzeichnis ................................................................................. XIX

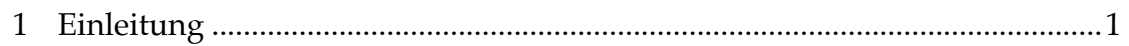

1.1 Problemstellung und Relevanz ..........................................................

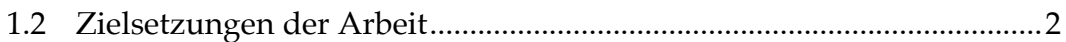

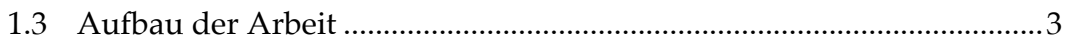

2 Theoretische Grundlagen ...............................................................................

2.1 Digital Business.................................................................................

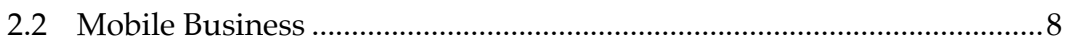

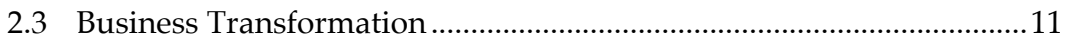

2.4 Maturity Models ............................................................................... 12

2.5 Business Engineering als Bezugsrahmen.............................................15

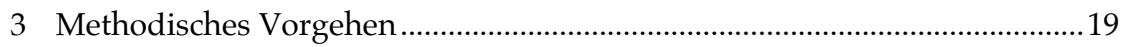

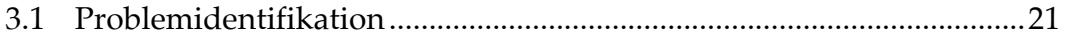

3.2 Existierende Maturity Models ..................................................................22

3.3 Entwicklung des Maturity Models ……………….................................24

3.4 Evaluation des Modells ............................................................................25

4 Existierende Maturity Models für Digital- und Mobile Business ..................22

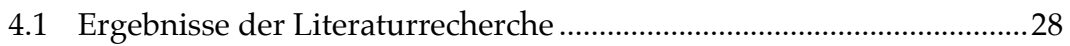

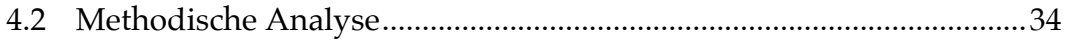

4.3 Inhaltliche Analyse..................................................................................

5 Entwicklung des Mobile Business Maturity Models ....................................... 43

5.1 Entwicklungsstrategie............................................................................. 
5.2 Struktur des Maturity Models ……………….........................................4

5.3 Auf der Literatur basierendes Maturity Model .......................................45

6 Evaluation des entwickelten Mobile Business Maturity Models ...................53

6.1 Untersuchungsziele und Interviewpartner ............................................53

6.2 Kritische Betrachtung der Evaluation ................................................55

6.3 Erkenntnisse aus den Experteninterviews.............................................57

6.4 Implikationen für das entwickelte Maturity Model................................59

7 Das Mobile Business Maturity Model und seine zehn Dimensionen............61

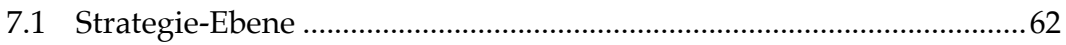

7.1.1 Bedeutung von Mobile Business ......................................................62

7.1.2 Organisation von Mobile Business ………………………….......... 65

7.1.3 Performance Management von Mobile Business ......................69

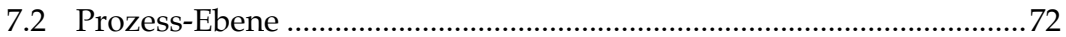

7.2.1 Kundenprozesse im Mobile Business..........................................73

7.2.2 Mitarbeiterprozesse mit Mobile Business ....................................76

7.2.3 Durchgängigkeit von Mobile Business ………………………......79

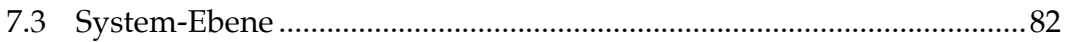

7.3.1 Technische Umsetzung von Mobile Business .............................82

7.3.2 Sicherheitsmechanismen für Mobile Business ............................86

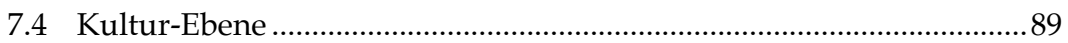

7.4.1 Kompetenzen zu Mobile Business ............................................89

7.4.2 Anwenderakzeptanz von Mobile Business .................................92

7.5 Gesamtbetrachtung des entwickelten Modells ....................................95

7.6 Transfer des Maturity Models und Transfermedien..............................100

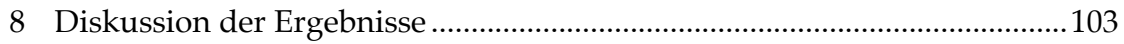

8.1 Reflexion des entwickelten Mobile Business Maturity Models..........103

8.2 Kritische Würdigung von Maturity Models im Allgemeinen ............105

9 Schlussfolgerungen und Diskussion ...............................................................107 
9.1 Zusammenfassung der Erkenntnisse

9.2 Implikationen für Theorie und Praxis...................................................108

9.3 Grenzen der Arbeit und Ausblick.......................................................109

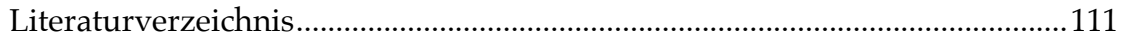

Verzeichnis der verwendeten Internet-Quellen .................................................121

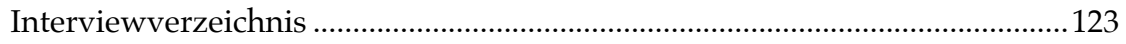

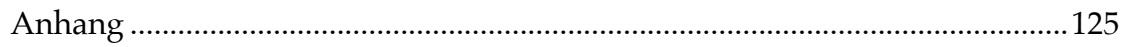

Das im Rahmen der Arbeit entwickelte Excel-basierte Assessment-Werkzeug zur Anwendung des Mobile Business Maturity Models kann direkt von http:/ / fyayc.com/mobile-maturity oder auf der Produktseite des Buches unter www.springer.com heruntergeladen werden. 


\section{Abbildungsverzeichnis}

Abbildung 1: Generischer Entwicklungsprozess von Maturity Models 15

Abbildung 2: St. Galler Business Engineering-Landkarte (Version 3.0).............17

Abbildung 3: Vorgehen zur Entwicklung des Maturity Models .........................20

Abbildung 4: Vorgehen bei der Literaturrecherche und -auswertung ...............22

Abbildung 5: Zusammenhang zwischen Innovationsreife und -diffusion ........48

Abbildung 6: Ziele der Untersuchung zur Evaluation des Maturity Models...53

Abbildung 7: Die zehn Dimensionen des Mobile Business Maturity Models ..61

Abbildung 8: Organisationstypen für Mobile Business ........................................68

Abbildung 9: Ausschnitte des Excel-basierten Assessment-Werkzeugs ..........101 


\section{Tabellenverzeichnis}

Tabelle 1: Ausgewählte Definitionen von Digital Business und E-Business........5

Tabelle 2: Ausgewählte Definitionen von Mobile Business..................................... 8

Tabelle 3: Übersicht über die geplante Literaturauswertung...............................27

Tabelle 4: Bestehende Maturity Models zu Digital- und Mobile Business........29

Tabelle 5: Methodische Analyse der gefundenen Maturity Models ....................35

Tabelle 6: Inhaltliche Analyse der gefundenen Maturity Models ........................38

Tabelle 7: Identifizierte Dimensionen aus der inhaltlichen Analyse....................39

Tabelle 8: Bestandteile des Maturity Models ........................................................... 44

Tabelle 9: Mobile Business Maturity Model vor der Evaluation ..........................46

Tabelle 10: Dimensionen und -objekte vor der Evaluation des Modells ............49

Tabelle 11: Befragte Experten zur Evaluation des Maturity Models....................54

Tabelle 12: Dimension 1.1 „Bedeutung von Mobile Business“ .............................63

Tabelle 13: Dimension 1.2 „Organisation von Mobile Business“..........................65

Tabelle 14: Dimension 1.3 „Performance Management von Mobile Business“ 69

Tabelle 15: Dimension 2.1 „Kundenprozesse mit Mobile Business“....................74

Tabelle 16: Dimension 2.2 „Mitarbeiterprozesse mit Mobile Business“ .............76

Tabelle 17: Dimension 2.3 „Durchgängigkeit von Mobile Business“ “.................79

Tabelle 18: Dimension 3.1 „Technische Umsetzung von Mobile Business“......83

Tabelle 19: Dimension 3.2 „Sicherheitsmechanismen für Mobile Business“ ....86

Tabelle 20: Dimension 4.1 „Kompetenzen zu Mobile Business“ ......................... 90

Tabelle 21: Dimension 4.2 „Anwenderakzeptanz von Mobile Business“.......... 93

Tabelle 22: Die fünf Reifegrade des Mobile Business Maturity Models ............ 96

Tabelle 23: Mobile Business Maturity Model in der finalen Version ...................98 


\section{Abkürzungsverzeichnis}

AG

BYOD

$\mathrm{CIO}$

COPE

CYOD

D

d.h.

Dr.

E

ERP

et al.

etc.

evtl.

GPS

$\mathrm{h}$

HSG

ICT

IKT

insb.

IT

Aktiengesellschaft

Bring Your Own Device (englisch für „Bring dein eigenes Gerät mit")

Chief Information Officer (englisch für Verantwortlicher für Informations- und Kommunikationstechnologie)

Corporate owned Personally Enabled (englisch für „Im Besitz des Unternehmens, für die persönliche Nutzung freigegeben“) Choose Your Own Device (englisch für „Wähle dein eigenes Gerät aus")

Deutschland

das heisst

Doktor(in)

Electronic (englisch für Elektronisch)

Enterprise Resource Planning

(englisch für Unternehmensressourcenplanung)

et alii (lateinisch für und andere)

et cetera (lateinisch für und so weiter)

eventuell

Global Positioning System

(englisch für Globales Positionsbestimmungssystem)

hour(s) (englisch für Stunde(n))

Universität St. Gallen

Information and Communication Technology

(englisch für Informations- und Kommunikationstechnologie)

Informations- und Kommunikationstechnologie

insbesondere

Informationstechnologie 
IWI-HSG Institut für Wirtschaftsinformatik der Universität St. Gallen

Kap. Kapitel

KMU Kleine(s) und mittlere(s) Unternehmen

KPI Key Performance Indicator (englisch für Leistungskennzahl)

M Mobile

MDM Mobile Device Management (englisch für Verwaltung von Mobilgeräten)

M\&A Mergers \& Acquisitions (englisch für Fusionen \& Übernahmen)

$\min \quad$ Minute(n)

MM Maturity Model

Nr. Nummer

PIN Persönliche Identifikationsnummer

Prof. Professor(in)

resp. respektive

S. Seite(n)

SME Small and Medium-sized Enterprise(s)

(englisch für kleine(s) und mittlere(s) Unternehmen)

St. Sankt

Vgl. Vergleiche

z.B. zum Beispiel

ZH Kanton Zürich 